Noname manuscript No.

(will be inserted by the editor)

\section{Stabilization and tracking control algorithms for VTOL aircraft: Theoretical and practical overview}

\author{
J. Betancourt • P. \\ Castillo • R. Lozano
}

Received: date / Accepted: date

\begin{abstract}
Control theory applied to multirotor aerial systems (MAS) has gained attention with the recent increase on the power computation for embedded systems. These systems are now able to perform the calculations needed for a variety of control techniques, with lower cost of sensors and actuators. These types of control algorithms are applied to the position and the attitude of MAS. In this paper, a brief overview evaluation of popular control algorithms for multirotor aerial systems, especially for VTOL - Vertical Take-Off and Landing aircraft, is presented. The main objective is to provide a unified and accessible analysis, placing the classical model of the VTOL vehicle and the studied control methods into a proper context. And therefore, to provide the basis for beginner users working in aerial vehicles. In addition, this work contributes in presenting a comprehensive analysis of the implementation for the Nonlinear and Linear Backstepping, Nested Saturation and the Hyperbolic Bounded Controllers. These techniques are selected and compared to evaluate the performance of the aircraft, by simulations and experimental studies.
\end{abstract}

Keywords Multirotor Aerial Systems · Classical approach · Virtual appraoch · Nonlinear/Linear Backstepping · Nested Saturation · Hyperbolic Bounded Control

J. Betancourt, P. Castillo and R. Lozano

Sorbonne Universits, Heudiasyc - UMR CNRS 7253

Université de technologie de Compiègne

CS 60319 - 57 avenue de Landshut 60203

COMPIEGNE CEDEX - FRANCE

E-mail: (gubetanc,castillo,rlozano)@hds.utc.fr

\section{Introduction}

Unmanned Aerial Vehicles systems (UAVs) have been studied extensively in recent years $[23,40]$, due to their high mobility and capacity to perform tasks with complete autonomy. Besides, these vehicles present an ideal test-bed for innovative theoretical approaches to the problem of controlling mechanical systems. They are characterized by their under-actuation, low computational power, high working frequency, and low autonomy [22]. Out of all UAVs, multirotors stand out for their good manoeuvrability, stability and payload. The first studies of these multirotors vehicles were based on stabilizing their attitude because it represents the main challenge before flying the vehicle. VTOL vehicles, specially the PTVOL - Vertical Take-Off and Landing or the Quadrotor configurations, are taken as particular cases of study and several control methodologies have been used to stabilize them, we can cite for example; Backstepping [54,47,17], Sliding Mode Control $[51,27,37]$, PID $[1,19,32,41,33]$, optimal control [26, 28, $10,45]$, robust control $[8,53,12]$, learning-based control [35], and others [3,55,31].

\subsection{Related work}

In control systems for multirotor aerial vehicles there are two different types of control, depending on the loop to which the controller is applied; the position controller and the attitude controller. In fact, there is no direct actuation control per se. Attitude control is the concept of pointing a craft in the desired direction. More specifically, it involves controlling the orientation of the robot on its three Euler angular velocities (roll rate $\dot{\theta}$, pitch rate $\dot{\phi}$ and yaw rate $\dot{\psi}$ ) and controlling the robots thrust in the Z-axis of the robot frame of reference. This is performed due to the underactuation characteristics of the robot. Then, the outputs of this control are sent to a mixing-of-motors algorithm to generate the reference signals for the actuators of the MAVs. In these cases, the attitude control algorithm must take into account the number of motors and their respective control signal. In contrast, position control is the controller that gives the Euler angles as references to the attitude controller in order for the MAV to move in a desired trajectory [25].

For the last 10 years, the two dynamics attitude and position were popularly controlled by monitoring the altitude ( $z$-axis) using feedback linearization followed by a linear/nonlinear controller to achieve the desired altitude. The values of the control torque can be founded by replacing the controlled altitude in the atti- 
tude expression. This method for designing the control torque will be denoted classical approach in this paper. In addition, adaptive control has been studied to solve the problems of stabilization and tracking of the dynamics. Hossein et al. proposed in [6] a novel trajectory tracking scheme by combining two inner feedback linearization loops to stabilize the nominal quadrotor. Moreover, Wenchao presented in [24] a robust adaptive tracking control scheme based on a self-tuning regulator. This tracking method is implemented in the inner loop and a classical proportional integrator (PI) controller is to be employed in the outer loop. In this way, Wenchao verified that the robustness of the whole system is guaranteed. Similarly, Tomashevich introduced the altitude and attitude control laws for the quadrotor based on the passivity method [44]; The simulation results demonstrate high efficiency and robustness with respect to the plant parametric uncertainty.

Furthermore, a time-varying backstepping technique is presented by Santiaguillo-Salinas et al. [39], where a $3 D$ control tracking law is studied and applied experimentally on a quadrotor. This control law is implemented by assuming that only the position and orientation parameters of the vehicles are known; whereas the linear and angular velocities are estimated using Luenberger observers. Rolsado-Serrano in [36] improved the work developed by Santiaguillo-Salinas by considering a non-linear time-varying version of the backstepping technique. This work takes all the non-linearities of the dynamics into account; resulting in a higher stability validated theoretically with numerical simulations.

Moreover, dynamic inversion is a new control technique developed in $[4,9]$ for the stabilization and dynamics tracking of a quadrotor vehicle. The tracking controller is composed of two loops; The dynamic inversion is implemented in the inner loop, where an internal dynamics stabilization is applied to the outer loop. The control objectives are enclosed in the form of constraint differential equation, and the resultant control law is obtained by inverting the constraint dynamics using Moore-Penrose Generalized Inverse (MPGI). Computer simulations were performed to exhibit the performance of the offered control technique in nominal and perturbed conditions. In addition to the dynamic inversion controllers, Antonio-Toledo et al. proposed in [5] an integral backstepping controller with sliding mode control approach for a quadrotor. In this approach the control of the UAV is performed in the altitude and position control. The altitude control produces translational force, which is used to calculate virtual controls for the $X$ and $Y$ movements and desired angles for pitch and roll. The proposed controller was tested in a Qball-
$\mathrm{X} 4$ prototype. In the same sense, J. Escareño and S. Salazar addressed a hierarchical control in [11], based on sliding-mode and adaptative control techniques, to deal with slow and fast time-varying wind conditions respectively. A backstepping technique is used to stabilize the inner-loop heading dynamics. Simulations results show the validity of the proposed control strategy while tracking a time-parametrized straight-line and sinusoidal trajectory. In addition, Ibarra-Jimenez and Castillo designed in [18] a controller that ensures the quick convergence in the dynamics of the quadrotor in order to catch a ball before it touches the ground. The algorithm is based on the sliding mode approach to assure robustness and finite time convergence. Simulations results verifies the performance of the controller to catch the ball even in presence of unknown and external disturbances.

Recently, a new fashion to design the torque control of the vehicle is considering the vehicle model fully actuated by means of a virtual input. In this paper, we will call the aforementioned as virtual approach. In this context, there are some works exploiting the differential flatness of the quadrotor model and then, with a trajectory generation algorithm, compute high-performance flight trajectories that are capable of moving a quadrotor from a large class of initial states to a given target point that will be reached at rest $[16,13,29]$. The algorithm can compute a feasible trajectory within tens of microseconds; the remaining computation time is used to iterative improve the trajectory. For example, Riu Wang proposed in [48] a control scheme based on a virtual control input using a backstepping approach with Nussbaum function, a priori-bounded control torque for the rotational subsystem was designed to track the desired orientations generated by the translational subsystem. In the same sense, Xu et al. addressed in [50] a robust cost controller derived by Lyapunov stability theorem. The designed robust guaranteed cost controller ensures the closed-loop system asymptotically stable and the robustness with respect to all admissible parameter uncertainties. Besides, Warier et al. solved in [49] the tracking control problem in two-step approach. First, a translational control scheme that tracks the desired position trajectory is constructed assuming the translational dynamics to be fully actuated. The magnitude of the translational control input is used as the magnitude of the control thrust. Second, the unit vector representing the direction of the translational control input used as the desired thrust direction.

This paper gives an overview focused on the two existing methods to design attitude and positions controllers to solve the stabilization and tracking problems 
that have been proposed in the time span from the first works in MAV control in 2002 to date. Our research has identified more than two hundred works, most of which are conference papers. We have selected for this document only journal papers and the most prominent recent conferences, so that the reader can understand the evolution of the state-of-the-art in multirotor control. Therefore, four of these control algorithms have be chosen to be implemented and compared. These control algorithms are described in detail and applied to a simulation model and validated through a serie of experimental tests. To the best of the authors knowledge, this is the first time that the virtual control algorithm based on hyperbolic bounded functions is implemented in real time experiments.

The remainder of the paper is structured as follows: The simplified dynamic model of a PVTOL vehicle is given in section 2 . We use this configuration because most multirotors with parallel motors can be seen as a generalization of the PVTOL vehicle. The control algorithms are introduced in section 3, details in their conception are given using the Lyapunov theory. Section 4 reports the numerical and experimental validation and Section 5 a discussion of the control schemes and their performances are given. Finally, section 6 details the conclusions of this work.

\section{The PVTOL aircraft}

As previously explained, the PVTOL aircraft is the most simple VTOL configuration, it represents the longitudinal model of the helicopter, the simplified model of a plane moving in the horizontal plane and the quadcopter can be seen as two PVTOL interconnected, as shown in Fig 8. It is an underactuated system and is composed by two independent motors that produce a force and a torque on the plane. Therefore, this configuration will be used to better illustrate the stability analysis of the algorithms. Moreover, the results can be expanded for other VTOL configurations.

The dynamical model is presented following these assumptions

- The vehicle structure is rigid and symmetrical,

- Its center of mass and $O_{B}$ (body frame) coincides,

- The pitch moment creates a force that is perpendicular to $z$ axis,

Let consider $\mathscr{I}=\left\{O_{I}, x_{I}, z_{I}\right\}$ an inertial frame and $\mathscr{B}=\left\{O_{B}, x_{B}, z_{B}\right\}$ a rigid frame attached to center of mass of the vehicle.

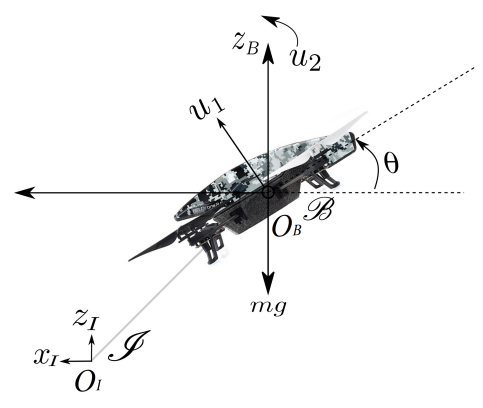

Fig. 1: Schematic model of the PVTOL aircraft.

The dynamics of a rigid body under external forces using the Newton-Euler formalism can be expressed as

$$
\begin{aligned}
\dot{\xi}(t) & =v(t), & m \dot{v}(t) & =R(t) F(t), \\
\dot{R}(t) & =R(t) \hat{\Omega}(t), & J \dot{\hat{\Omega}} & =-\Omega(t) \times J \Omega(t)+\tau(t)
\end{aligned}
$$

where $\xi(t)$ denotes the position of the vehicle with respect to the frame $\mathscr{I}, v(t) \in \mathscr{I}$ describes the linear velocity, $\Omega(t)$ represents the angular velocity of the body defined in $\mathscr{B}$, and $m$ is the total mass of the vehicle. The constant moment of inertia is denoted by $J$ expressed in $\mathscr{B}, \tau(t)$ expresses the torques applied in the rigid body, $\hat{\Omega}(t)$ introduces the skew-symmetric matrix of $\Omega(t), R(t)$ means the rotation matrix from $\mathscr{B}$ to $\mathscr{I}$, and $F(t)$ are the forces applied to the rigid body.

From (1) and Figure 8, the nonlinear dynamic model of a PTVOL aircraft can be represented as

$$
\begin{aligned}
m \ddot{x} & =-u_{1} \sin \theta+\epsilon u_{2} \cos \theta \\
m \ddot{z} & =u_{1} \cos \theta+\epsilon u_{2} \sin \theta-m g \\
J \ddot{\theta} & =u_{2}
\end{aligned}
$$

where $x$ and $z$ are respectively the lateral and vertical coordinates of the aircraft center of mass, $\theta$ denotes the pitch angle with respect to the horizon, $u_{1}$ describe the main thrust composed by the forces produced by each motor $f_{i}, u_{2}$ represent the pitching moment and $g$ corresponds to the acceleration due to gravity. $\epsilon$ represents the coupling value between the torque and the lateral force, in general, it is not well known and in several cases neglected. Thus, the simplified nonlinear model for the PVTOL with $\epsilon=0$ can be described in the following form

$$
\begin{aligned}
m \ddot{x} & =-u_{1} \sin \theta \\
m \ddot{z} & =u_{1} \cos \theta-m g \\
J \ddot{\theta} & =u_{2}
\end{aligned}
$$

Note that in [34], it was proved that by an appropriate change of coordinates, (2)-(4) can be represented as (5)(7) without neglecting the term $\epsilon$. From the above, the 
input vector can be defined as

$\boldsymbol{u}=\left[\begin{array}{l}u_{1}(t) \\ u_{2}(t)\end{array}\right]=\left[\begin{array}{cc}1 & 1 \\ l & -l\end{array}\right]\left[\begin{array}{l}f_{1}(t) \\ f_{2}(t)\end{array}\right]$.

where $l$ denotes the distance between one rotor to the center of mass of the vehicle and $f_{1}$ and $f_{2}$ are the forces produced by motor 1 and 2 respectively.

\section{Control architectures}

Typically in the literature when controlling a VTOL vehicle is assumed the $z$-axis can be controlled by some linear or nonlinear controller using specifically $u_{1}$. Usually, this controller is based on a feedback linearization technique as presented in $[30,14,15,42]$. This classical procedure is often used because the idea is to guarantee the vehicle keeps at hover, and later control their displacement in the plane $x$ or $y$ making an under-actuated subsystem.

Following these ideas, the controller for (6) is very often proposed as

$u_{1}=\frac{m}{\cos \theta}\left(g+r_{1}\right)$

where $r_{1}$ is always designed to achieve $z \rightarrow z_{d}$, for instance $r_{1}=-k_{1}\left(z-z_{d}\right)-k_{2} \dot{z}$, where $k_{1}, k_{2}$ represent positive constants and $z_{d}$ denotes the desired altitude. Introducing (9) into (5)-(6), it follows

$$
\begin{aligned}
m \ddot{x} & =-\left(-k_{2} \dot{z}-k_{1}\left(z-z_{d}\right)+g\right) m \tan \theta \\
\ddot{z} & =-k_{2} \dot{z}-k_{1}\left(z-z_{d}\right)
\end{aligned}
$$

Note from (11), $\exists$ a time $T$ large enough such that for $t>T, z$ and $\dot{z}$ are arbitrarily small such that (10) can be expressed as

$\ddot{x} \approx-g \tan \theta$

For simplifying further analysis, consider $J=1$. Then rewriting (12) and (7), it yields

$$
\begin{aligned}
& \dot{x}_{1}=x_{2} \\
& \dot{x}_{2}=-g \tan \theta_{1} \\
& \dot{\theta}_{1}=\theta_{2} \\
& \dot{\theta}_{2}=u_{2}
\end{aligned}
$$

where $x_{1}=x, x_{2}=\dot{x}, \theta_{1}=\theta, \theta_{2}=\dot{\theta}$.

Notice that (13) describes an under-actuated subsystem representing the longitudinal dynamics of a VTOL vehicle. These dynamics will be used for conceiving three control algorithms using two different techniques; the backstepping and the saturation functions. These methodologies will be referred in the following as classical control inputs.

\subsection{Nonlinear Backstepping algorithm}

Define the error $e_{1}$ as $e_{1}=x_{1}-x_{1}^{d}$, where $x_{1}^{d}$ is the reference. Let us propose the following positive function $V_{1}=\frac{k_{1}}{2} e_{1}^{2}$, with $k_{1}>0$ denoting a constant, then taking the derivative with respect to time and proposing $x_{2}^{v}=$ $x_{2}^{d}-e_{1}$ as a virtual control input, it follows that

$\dot{V}_{1}=k_{1} e_{1}\left(x_{2}-x_{2}^{v}-e_{1}\right)=-k_{1} e_{1}^{2}+k_{1} e_{1}\left(x_{2}-x_{2}^{v}\right)$

where $\dot{x}_{1}^{d}=x_{2}^{d}$. Define the error $e_{2}=x_{2}-x_{2}^{v}$ thus, the previous yields $\dot{V}_{1}=-k_{1} e_{1}^{2}+k_{1} e_{1} e_{2}$. Propose the second positive function $V_{2}=\frac{k_{2}}{2} e_{2}^{2}$ with $k_{2}>0$ constant. Taking its derivative with respect to time and proposing $\delta_{1}^{v}=-\dot{x}_{2}^{v}+\frac{k_{1}}{k_{2}} e_{1}+e_{2}$ representing a second virtual control input, implies that

$\dot{V}_{2}=-k_{2} e_{2}^{2}-k_{1} e_{1} e_{2}+k_{2} e_{2}\left(\delta_{1}^{v}-g \tan \theta_{1}\right)$

Defining the error $e_{3}=\delta_{1}^{v}-g \tan \theta_{1}, \dot{V}_{2}$ yields

$\dot{V}_{2}=-k_{2} e_{2}^{2}-k_{1} e_{1} e_{2}+k_{2} e_{2} e_{3}$

Propose the third positive function $V_{3}=\frac{k_{3}}{2} e_{3}^{2}$, with $k_{3}>0$ constant. Differentiating $V_{3}$ and defining $\delta_{2}^{v}=$ $\dot{\delta}_{1}^{v}+\frac{k_{2}}{k_{3}} e_{2}+e_{3}$, it follows that

$\dot{V}_{3}=k_{3} e_{3}^{2}-k_{2} e_{3} e_{2}+k_{3} e_{3}\left(\delta_{2}^{v}-g\left(1+\tan ^{2} \theta_{1}\right) \theta_{2}\right)$

Defining the error $e_{4}=\delta_{2}^{v}-g\left(1+\tan ^{2} \theta_{1}\right) \theta_{2}$, then the above yields

$\dot{V}_{3}=-k_{3} e_{3}^{2}-k_{2} e_{3} e_{2}+k_{3} e_{3} e_{4}$

Proposing $V_{4}=\frac{k_{4}}{2} e_{4}^{2}$ as positive function, with $k_{4}>0$ defining a constant, and taking its derivative, it appears that

$\dot{V}_{4}=k_{4} e_{4}\left(\dot{\delta}_{2}^{v}-g\left(1+\tan ^{2} \theta_{1}\right)\left(u_{2}+2 \theta_{2}^{2} \tan \theta_{1}\right)\right)$

Proposing a control law given by

$u_{2}=\frac{1}{g\left(1+\tan ^{2} \theta_{1}\right)}\left(\dot{\delta_{2}^{v}}+\frac{k_{3}}{k_{4}} e_{3}+e_{4}\right)-2 \theta_{2}^{2} \tan \theta_{1}$

implies that $\dot{V}_{4}=-k_{4} e_{4}^{2}-k_{3} e_{4} e_{3}$.

Finally, define the following candidate Lyapunov function with the form $V_{T}=V_{1}+V_{2}+V_{3}+V_{4}$. Therefore

$\dot{V}_{T}=-k_{1} e_{1}^{2}-k_{2} e_{2}^{2}-k_{3} e_{3}^{2}-k_{4} e_{4}^{2} \leq 0$.

Expressing (14) with respect to the state variables, it follows that

$$
\begin{aligned}
u_{2}=\frac{1}{g\left(1+\tan ^{2} \theta_{1}\right)} & \left(\dddot{x}_{2}^{d}-4 \ddot{x}_{2}^{d}-\bar{k}_{1} \dot{x}_{2}^{d}+\bar{k}_{2}\left(x_{2}-x_{2}^{d}\right)\right. \\
& +\bar{k}_{3}\left(x_{1}-x_{1}^{d}\right)-\bar{k}_{4} g \tan \theta_{1} \\
& \left.-4 g\left(1+\tan ^{2} \theta_{1}\right) \theta_{2}\right)-2 \theta^{2} \theta_{1}
\end{aligned}
$$


where

$$
\begin{array}{ll}
\bar{k}_{1}=\bar{k}_{4}=k_{\theta}+6, & \bar{k}_{2}=2 k_{\theta}+4 \\
\bar{k}_{3}=k_{\theta}+\frac{k_{3} k_{1}}{k_{4} k_{2}}+1, & k_{\theta}=\frac{k_{1}}{k_{2}}+\frac{k_{2}}{k_{3}}+\frac{k_{3}}{k_{4}}
\end{array}
$$

\subsection{Control algorithm based on nested saturation}

The following control algorithm uses saturation functions to impose a bound in the control input. The first methodology was proposed by [43] designing the controller with nested saturation functions for linear systems and applied to nonlinear systems. Nevertheless after analysis, it can be observed that the controller structure using saturation functions imposes bounds in each state, such that, the following inequality can be applied $\tan \theta \approx \theta$, see [38,7]. From this assumption, (13) can be rewritten as follows

$$
\begin{aligned}
& \dot{x}_{1}=x_{2} \\
& \dot{x}_{2} \approx-g \theta_{1} \\
& \dot{\theta}_{1}=\theta_{2} \\
& \dot{\theta}_{2}=u_{2}
\end{aligned}
$$

A controller stabilizing (17) can be denoted by

$u_{2}=-\sigma_{a}\left(\theta_{2}+\sigma_{b}(\cdot)\right)$

where $\sigma_{i}$ defines a generalized saturation function such that $\left|\sigma_{i}(\cdot)\right| \leq i$ for positive constant $i: a, b, c, d$. The argument in $\sigma_{b}(\cdot)$ will be defined later to assure convergence of the states. Let us define a positive function $V_{1}=\frac{1}{2} \theta_{2}^{2}$, then its derivative is defined by

$\dot{V}_{1}=\theta_{2} \dot{\theta_{2}}=\theta_{2} u_{2}=-\theta_{2} \sigma_{a}\left(\theta_{2}+\sigma_{b}(\cdot)\right)$

if $\left|\theta_{2}\right| \geq b \Rightarrow \dot{V}_{1} \leq 0$. Then, $\exists t_{1}$ such that for $t \geq t_{1}$ $\left|\theta_{2}(t)\right| \leq b$, implying that $\left|\theta_{2}+\sigma_{b}(\cdot)\right| \leq 2 b$. Choosing $a \geq 2 b$, then (18) can be rewritten $\forall t>t_{1}$ as

$u_{2}=-\theta_{2}-\sigma_{b}(\cdot)$.

Define $\nu_{1}=\theta_{1}+\theta_{2}$, then, $\dot{\nu}_{1}=-\sigma_{b}(\cdot)$. Propose a positive function $V_{2}=\frac{1}{2} \nu_{1}^{2}$ and imposing $\sigma_{b}(\cdot)=\sigma_{b}\left(\nu_{1}+\right.$ $\left.\sigma_{c}(\cdot)\right)$ then, its derivative is determined by

$\dot{V}_{2}=\nu_{1} \dot{\nu}_{1}=-\nu_{1} \sigma_{b}\left(\nu_{1}+\sigma_{c}(\cdot)\right)$

if $\left|\nu_{1}\right| \geq c \Rightarrow \dot{V}_{2} \leq 0$. Then, $\exists t_{2} \geq t_{1}$ such that for $t \geq t_{2}$ $\left|\nu_{1}(t)\right| \leq c$ and $\left|\nu_{1}+\sigma_{c}(\cdot)\right| \leq 2 c$. Choosing $b \geq 2 c$, then (20) can be rewritten $\forall t>t_{2}$ as

$u_{2}=-\theta_{2}-\nu_{1}-\sigma_{c}(\cdot)$.
Define $\nu_{2}=\nu_{1}+\theta_{1}-x_{2} / g=\theta_{2}+2 \theta_{1}-x_{2} / g$, thus $\dot{\nu}_{2}=-\sigma_{c}(\cdot)$. Propose the positive function $V_{3}=\frac{1}{2} \nu_{2}^{2}$ with $\sigma_{c}(\cdot)=\sigma_{c}\left(\nu_{2}+\sigma_{d}(\cdot)\right)$, then its derivative is described by

$\dot{V}_{3}=\nu_{2} \dot{\nu}_{2}=-\nu_{2} \sigma_{c}\left(\nu_{2}+\sigma_{d}(\cdot)\right)$

if $\left|\nu_{2}\right| \geq d \Rightarrow \dot{V}_{3} \leq 0$. Then, $\exists t_{3} \geq t_{2}$ such that $\forall t \geq t_{3}$ $\left|\nu_{2}(t)\right| \leq d$ and $\left|\nu_{2}+\sigma_{d}(\cdot)\right| \leq 2 d$. Choosing $c \geq 2 d$, then (22) can be rewritten $\forall t>t_{3}$ as

$u_{2}=-\theta_{2}-\nu_{1}-\nu_{2}-\sigma_{d}(\cdot)$.

Define $\nu_{3}=\theta_{2}+3 \theta_{1}-3 \frac{x_{2}}{g}-\frac{x_{1}}{g}$ then $\dot{\nu}_{3}=-\sigma_{d}(\cdot)$. Propose $V_{4}=\frac{1}{2} \nu_{3}^{2}$ and $\sigma_{d}(\cdot)=\sigma_{d}\left(\nu_{3}\right)$, taking the derivative of $V_{4}$, it follows that

$\dot{V}_{4}=\nu_{3} \dot{\nu}_{3}=-\nu_{3} \sigma_{d}\left(\nu_{3}\right) \leq 0$

The previous implies that $\nu_{3} \rightarrow 0$, then from (23) it follows that $\nu_{2} \rightarrow 0$. From (21) implies that $\nu_{1} \rightarrow 0$, similarly from (19), $\theta_{2} \rightarrow 0$. From definition of $\nu_{1}$ it follows that $\theta_{1} \rightarrow 0$. From definition of $\nu_{2}$ this implies that $x_{2} \rightarrow 0$. And finally, from definition of $\nu_{3}$ it can be deduced that $x_{1} \rightarrow 0$.

Rewriting $u_{2}$, it yields

$$
\begin{aligned}
u_{\theta}= & -\sigma_{a}\left(\theta_{2}+\sigma_{b}\left(\theta_{1}+\theta_{2}+\sigma_{c}\left(\theta_{2}+2 \theta_{1}+x_{2}\right.\right.\right. \\
& \left.\left.\left.+\sigma_{d}\left(\phi_{2}+3 \phi_{1}-3 \frac{x_{2}}{g}-\frac{x_{1}}{g}\right)\right)\right)\right)
\end{aligned}
$$

\subsection{Linear Backstepping controller}

This controller is also useful when assuming that the vehicle is moving with small angles. The procedure is similar that for the nonlinear backstepping, nevertheless the obtained algorithm is quite different and has the form of a PD controller. For conceiving the algorithm a linear model is necessary, for our study, system (17) will be used.

Rewriting first equation of (17)

$\dot{x}_{1}=x_{2}$

propose $V_{1}=\frac{1}{2} x_{1}^{2}$, then $\dot{V}_{1}=x_{1} x_{2}$. if $x_{2} \rightarrow-k_{1} x_{1}$ with $k_{1}$ is a positive constant, then $\dot{V}_{1}=-k_{1} x_{1}^{2}$. Define the following error with the form

$\zeta_{2}=x_{2}-x_{2}^{v}$

with $x_{2}^{v}=-k_{1} x_{1}=\alpha_{1}$ defining the first virtual input. Rewriting (27) and the second equation of (17) with 28 and using $x_{1}=\zeta_{1}$

$\dot{\zeta}_{1}=\zeta_{2}+\alpha_{1}$

$\dot{\zeta}_{2}=-g \theta_{1}-\dot{\alpha}_{1}$ 
Define a positive definite function $V_{2}=V_{1}+{\frac{\zeta_{2}}{2}}^{2}$, then

$\dot{V}_{2}=\dot{V}_{1}+\zeta_{2} \dot{\zeta}_{2}=\dot{V}_{1}+\zeta_{2}\left(-g \theta_{1}-\dot{\alpha}_{1}\right)$

if $\left(-g \theta_{1}-\dot{\alpha}_{1}\right) \rightarrow-k_{2} \zeta_{2}$ with $k_{2}$ is a positive constant, then, $\dot{V}_{2}=\dot{V}_{1}-k_{2} \zeta_{2}^{2}$. Define the error

$\zeta_{3}=-g \theta_{1}-\alpha_{2}$

with $\alpha_{2}=k_{2} \zeta_{2}-\dot{\alpha}_{1}$ denoting the second virtual input. Rewriting (29) with the previous equation, it follows that

$\dot{\zeta}_{1}=\zeta_{2}+\alpha_{1}$

$\dot{\zeta}_{2}=-g \theta_{1}-\dot{\alpha}_{1}$

$\dot{\zeta}_{3}=-g \theta_{2}-\dot{\alpha}_{2}$

Define the positive definite function $V_{3}=V_{2}+\frac{\zeta_{3}^{2}}{2}$, then

$\dot{V}_{3}=\dot{V}_{2}+\zeta_{3} \dot{\zeta}_{3}=\dot{V}_{2}+\zeta_{3}\left(-g \theta_{2}-\dot{\alpha}_{2}\right)$

if $\left(-g \theta_{2}-\dot{\alpha}_{2}\right) \rightarrow-k_{3} \zeta_{3}$, with $k_{3}$ is a positive constant, then $\dot{V}_{3}=\dot{V}_{2}-k_{3} \zeta_{3}^{2}$. Define the error

$\zeta_{4}=-g \theta_{2}-\alpha_{3}$

where $\alpha_{3}=\left(g \theta_{2}\right)^{v}=k_{3} \zeta_{3}-\dot{\alpha}_{2}$ is the third virtual input. Rewriting (32) with $\dot{\zeta}_{4}$, it follows that

$\dot{\zeta}_{1}=\zeta_{2}+\alpha_{1}$

$\dot{\zeta}_{2}=-g \theta_{1}-\dot{\alpha}_{1}$

$\dot{\zeta}_{3}=-g \theta_{2}-\dot{\alpha}_{2}$

$\dot{\zeta}_{4}=-g u_{2}-\dot{\alpha}_{3}$

Propose the Lyapunov candidate function $V_{4}=V_{3}+\frac{\zeta_{4}^{2}}{2}$, then

$\dot{V}_{4}=\dot{V}_{3}+\zeta_{4} \dot{\zeta}_{4}=\dot{V}_{3}+\zeta_{4}\left(-g u_{2}-\dot{\alpha}_{3}\right)$

Propose the control input

$u_{2}=\frac{1}{g}\left(k_{4} \zeta_{4}-\dot{\alpha}_{3}\right)$

with $k_{4}>0$ is a constant. Then

$\dot{V}_{4}=\dot{V}_{3}-k_{4} \zeta_{4}^{2}=-k_{1} \zeta_{1}^{2}-k_{2} \zeta_{2}^{2}-k_{3} \zeta_{3}^{2}-k_{4} \zeta_{4}^{2}<0$

The above implies system (35) goes to zero implying that (17) is globally asymptotically stable. Rewriting (37) with respect to the state variables, it follows that

$u_{2}=-\frac{\bar{k}_{1}}{g} x_{1}-\frac{\bar{k}_{2}}{g} x_{2}+\bar{k}_{3} \theta_{1}+\bar{k}_{4} \theta_{2}$

where

$\bar{k}_{1}=k_{1} k_{2} k_{3} k_{4}$

$\bar{k}_{2}=k_{1} k_{2}\left(k_{3}+k_{4}\right)+k_{3} k_{4}\left(k_{1}+k_{2}\right)$

$\bar{k}_{3}=k_{1}\left(k_{2}+k_{3}+k_{4}\right)+k_{2}\left(k_{3}+k_{4}\right)+k_{3} k_{4}$

$\bar{k}_{4}=k_{1}+k_{2}+k_{3}+k_{4}$
3.4 Fully actuated approach

Previous approaches, as explained, use mainly the control input $u_{1}$ to stabilize the altitude of a VTOL while $u_{2}$ is necessary to control the underactuated subsystem. Some works in literature propose to rewrite the system constructing a cascade structure which only consists of two fully actuated subsystems by means of a virtual input. Motivated by the works developed by [46,2], we propose simple smooth bounded controllers that can easily be implemented in VTOL aircrafts with parallel motors for tracking set-points and time-varying trajectories.

The methodology is explained as follows; propose a vector control input, $F$, containing the virtual control laws to stabilize the translational states in the VTOL vehicle. These virtual control inputs will be related with a desired orientation matrix, $R_{d}$, of the vehicle and with its main control input, $u$, as follows

$F=u R_{d}$

In our case of study, the virtual control laws will be designed for the vertical and longitudinal axis of the PVTOL with the form $F=\left[\begin{array}{ll}F_{1} & F_{2}\end{array}\right]^{T}$, similarly $R_{d} \in$ $\mathbb{R}^{2 \times 1}$, is defined as

$$
R_{d}=\left[\begin{array}{l}
\sin \theta_{d} \\
\cos \theta_{d}
\end{array}\right]
$$

Next step, is to rewrite the original dynamic system, in our case it is represented by equations (5)-(7). Thus the new system is described as

$$
\begin{aligned}
m \ddot{q} & =u_{1} R+m g e_{2} \\
J \ddot{\theta} & =u_{2}
\end{aligned}
$$

where $q=\left[\begin{array}{ll}x & z\end{array}\right]^{T}, R=\left[\begin{array}{ll}\sin \theta & \cos \theta\end{array}\right]^{T}$, and $e_{2}=\left[\begin{array}{ll}0 & 1\end{array}\right]^{T}$. Notice that in (41), $u$ is the main control input, in our case, $u=u_{1}$. Therefore, using (41) into first equation of $(42)$

$$
\begin{aligned}
m \ddot{q} & =u_{1} R+m g e_{2}+u_{1} R_{d}-u_{1} R_{d} \\
& =u_{1}\left(R-R_{d}\right)+m g e_{2}+u_{1} R_{d}
\end{aligned}
$$

Define $\omega=u_{1}\left(R-R_{d}\right)$, thus

$m \ddot{q}=\omega+m g e_{2}+F$.

The desired orientation can be found using (41), then

$\theta_{d}=\tan \left(\frac{F_{1}}{F_{2}}\right)^{-1}$ 
Notice from (41) that $R_{d}$ is an orthogonal vector, therefore

$u_{1}=\sqrt{F_{1}^{2}+F_{2}^{2}}$

Define the control objective

$\lim _{t \rightarrow \infty}\left\|q(t)-q_{d}(t)\right\|=0$.

where $q_{d}=\left[\begin{array}{ll}x_{d} & y_{d}\end{array}\right]^{T}$ are the longitudinal and vertical references. Denoting the tracking error as $p=\left[\begin{array}{ll}p_{1} & p_{2}\end{array}\right]$ such that

$p_{1}=q-q_{d} \quad p_{2}=\dot{q}-\dot{q}_{d}$

Differentiating (47) and by means of (43)

$\dot{p_{1}}=p_{2}$

$\dot{p_{2}}=\frac{\omega}{m}-g e_{2}+\frac{F}{m}-\ddot{q}_{d}$.

where $\ddot{q}_{d}$ means the desired acceleration.

\subsubsection{Control based on hyperbolic functions}

Let us propose $F$ in (48) as

$F=m\left(-\sigma_{1}\left(A p_{1}+B p_{2}\right)-\sigma_{2}\left(B p_{2}\right)+g e_{2}+\ddot{q}_{d}\right)$

where $A, B \in \mathbb{R}^{2 \times 2}$ are diagonal positive matrices constant, $\sigma_{i}$ signifies a saturation function with the form $\sigma(\varsigma)=\bar{\sigma} \tanh (\varsigma)$ and $\bar{\sigma}$ means a bounded constant.

Hence, the bounded control $F$ in (49) makes system (48) globally asymptotically stable.

Proof: First, to prove the asymptotic convergence of solution $p(t)$ to the origin, observe from (41) and (43) that $\|\omega\| \leq 2 \bar{u}$. This implies that the closed-loop system using (48) and (49) satisfies the Lipschitz condition ${ }^{1}$, and hence has a unique solution over $[0, T]$ with $T \geq 0$. Moreover, if $T$ is bounded, then $p(t)$ is bounded $\forall t \in$ $[0, T]$. This proof is inspired in $[52]$.

Furthermore, if $\lim _{t \rightarrow \infty}\|\omega(t)\|=0$ then $\lim _{t \rightarrow \infty}\|p(t)\|=$ $0 \forall p(0)$, that is, for every $\epsilon_{1}$, for every $p(0)$, there exists $\epsilon_{2}, T_{\epsilon_{1}}, T_{\epsilon_{2}}>0$ and $T_{\epsilon_{1}} \geq T_{\epsilon_{2}}$ such that

$\|\omega(t)\|<\epsilon_{2}, \forall t \geq T_{\epsilon_{2}} \Rightarrow\|p(t)\|<\epsilon_{1}, \forall t \geq T_{\epsilon_{1}}$.

Towards this end, inspired by the work developed by [46], let us propose the following candidate Lyapunov function

$V_{1}(p)=\sum_{i=1}^{2} \int_{0}^{\left(a_{i} p_{1 i}+b_{i} p_{2 i}\right)} \sigma_{1 i}\left(\varsigma_{i}\right) d \varsigma_{i}+\frac{1}{2} p_{2}^{T} A p_{2}$

\footnotetext{
${ }^{1}$ See [20], Theorem 3.1 and page 446
}

where $a_{i}, p_{1 i}, b_{i}, p_{2 i}, \sigma_{1 i}$ and $\varsigma_{i}$ for $i=1,2$ are the elements of, $A, B, p_{1}, p_{2}, \sigma_{1}$ and $\varsigma$ respectively. The time derivative of $V_{1}(p)$ along (48) and (49) is

$$
\begin{aligned}
\dot{V}_{1}= & \dot{p}_{1}^{T} A \sigma_{1}\left(A p_{1}+B p_{2}\right)+{\dot{p_{2}}}^{T} B \sigma_{1}\left(A p_{1}+B p_{2}\right) \\
& +p_{2}^{T} A \dot{p_{2}} \\
= & -\sigma_{1}\left(A p_{1}+B p_{2}\right) B \sigma_{1}\left(A p_{1}+B p_{2}\right)-\sigma_{2}\left(B p_{2}\right) B \\
& \sigma_{1}\left(A p_{1}+B p_{2}\right)+\frac{\omega^{T}}{m} B \sigma_{1}\left(A p_{1}+B p_{2}\right) \\
& -\sigma_{2}\left(B p_{2}\right) A p_{2}+\frac{\omega^{T}}{m} A p_{2} \\
& \leq-\lambda_{\min }(B)\left\|\sigma_{1}\left(A p_{1}+B p_{2}\right)\right\|^{2}+\lambda_{\max }(B) \\
& \left\|\sigma_{1}\left(A p_{1}+B p_{2}\right)\right\|\left\|\sigma_{2}\left(B p_{2}\right)\right\|-\lambda_{\min }(A) p_{2}^{T} \sigma_{2}\left(B p_{2}\right) \\
& +\frac{\omega^{T}}{m}\left(A p_{2}+B \sigma_{1}\left(A p_{1}+B p_{2}\right)\right)
\end{aligned}
$$

Using Young's inequality ${ }^{1}$, the second term of (52), satisfies

$$
\begin{aligned}
& \lambda_{\max }(B)\left\|\sigma_{1}\left(A p_{1}+B p_{2}\right)\right\|\left\|\sigma_{2}\left(B p_{2}\right)\right\| \\
& \leq \frac{\lambda_{\max }(B)}{2}\left\|\sigma_{1}\left(A p_{1}+B p_{2}\right)\right\|^{2} \\
& \quad+\frac{\lambda_{\max }(B)}{2}\left\|\sigma_{2}\left(B p_{2}\right)\right\|^{2}
\end{aligned}
$$

Furthermore, there exists $\nu>0$ such that \|\|$\sigma_{2}\left(B p_{2}\right) \leq$ $\nu \lambda_{\max }(B)\left\|p_{2}\right\|$, for all $p_{2}$, then the third term of $(52)$ satisfies

$\left\|\sigma_{2}\left(B p_{2}\right)\right\|^{2} \leq \nu \lambda_{\max }(B) p_{2}^{T} \sigma_{2}\left(B p_{2}\right)$

Substituting (4) into (53), yields

$$
\begin{aligned}
\left\|\sigma_{1}\left(A p_{1}+B p_{2}\right)\right\|\left\|\sigma_{2}\left(B p_{2}\right)\right\| & \leq \frac{\left\|\sigma_{1}\left(A p_{1}+B p_{2}\right)\right\|^{2}}{2} \\
& +\frac{\nu \lambda_{\max }}{2} p_{2}^{T} \sigma_{2}\left(B P_{2}\right)
\end{aligned}
$$

From (55), the derivative $\dot{V}_{1}$ in (52) satisfies

$\dot{V}_{1} \leq-W_{1}(p, \omega)$

where

$$
\begin{aligned}
W_{1}(p, \omega)= & c_{1}\left\|\sigma_{1}\left(A p_{1}+B p_{2}\right)\right\|^{2}+c_{2} p_{2}^{T} \sigma_{2}\left(B p_{2}\right) \\
& -\frac{\|\omega\|}{m}\left(\lambda_{\max }(A)\left\|p_{2}\right\|+c_{3}\right)
\end{aligned}
$$

with $c_{1}=\frac{2 \lambda_{\min }(B)-\lambda_{\max }(B)}{2}, c_{2}=\frac{2 \lambda_{\min }(A)-\nu \lambda_{\max }^{2}(B)}{2}$, $c_{3}=\lambda_{\max }(B) \overline{\sigma_{1}}$ with $\overline{\sigma_{1}} \geq\left\|\sigma_{1}(\cdot)\right\|$. In order to ensure $c_{1}, c_{2}, c_{3}>0$, the matrices $A, B$ are chosen as follows:

$2 \lambda_{\min }(B)>\lambda_{\max } B ; \quad 2 \lambda_{\min }(A)>\nu \lambda_{\max }(B)^{2}$

(56) is used to get (50). Let the ball $\mathcal{B}_{\epsilon_{1}}=\left\{p \in \mathbb{R}^{3} \mid\|p\| \leq\right.$ $\left.\epsilon_{1}\right\}$, and show that $p(t)$ approaches $\mathcal{B}_{\epsilon_{1}}$ after the time 
$T_{\epsilon_{1}}$. Let $\alpha_{1}=\min _{\|p\|=\epsilon_{1}} V_{1}(p)$, then $\alpha_{1}>0$. Take $\beta_{1} \in$ $\left(0, \alpha_{1}\right)$ and define the set $\Omega_{\beta_{1}}=\left\{p \in \mathcal{B} \mid V_{1}(p) \leq \beta_{1}\right\}$, then $\Omega_{\beta_{1}}$ is in the interior of $\mathcal{B}_{\epsilon_{1}}$. As $V_{1}(p)$ is continuous and $V_{1}(0)=0$, there exists $\gamma_{1}$ such that $0<\gamma_{1}<\epsilon_{1}$ and $\beta_{2}=\max _{\|p\|=\gamma_{1}} V_{1}(p)<\beta_{1}$. Let the ball $\beta_{\gamma_{1}}=\{p \in$ $\left.\Omega_{\beta_{1}} \mid\|p\| \leq \gamma_{1}\right\}$, and take $\alpha_{2}=\min _{\|p\|=\gamma_{1}} V_{1}(p)$, then we have $0<\alpha_{2}<\beta_{2}<\beta_{1}<\alpha_{1}$. Consequently, let the ball $\beta_{\gamma_{2}}=\left\{p \in \mathcal{B}_{\gamma_{1}} \mid\|p\| \leq \gamma_{2}\right\}$ where $\gamma_{2}$ satisfies $0<$ $\gamma_{2}<\gamma_{1}$ and $0<\min _{\|p\|=\gamma_{2}} V_{1}(p) \leq \max _{\|p\|=\gamma_{2}} V_{1}(p)<$ $\alpha_{2}$, then from the above construction we have

$\mathcal{B}_{\gamma_{2}} \subset \mathcal{B}_{\gamma_{1}} \subset \Omega_{\beta_{1}} \subset \mathcal{B}_{\epsilon_{1}}$

and the Lyapunov function $V_{1}(p)$ satisfies

$$
\begin{aligned}
\max _{\|p\|=\gamma_{2}} V_{1}(p) & <\min _{\|p\|=\gamma_{1}} V_{1}(p) \\
& <\max _{\|p\|=\gamma_{1}} V_{1}(p)<\beta_{1}<\alpha_{1} .
\end{aligned}
$$

In view of (59) it is sufficient to show that $p(t)$ approches $\mathcal{B}_{\gamma_{2}}$ after the finite time $T_{\epsilon_{1}}$. Then, the proof is completed.

As the PVTOL is composed by mechanical and electrical parts, constraints are needed in order to respect the limits of its actuators and mechanical movements. Using (45) and (49) it yields

$$
u^{2}=F_{1}^{2}+F_{2}^{2}
$$$$
u^{2} \leq\left(\sigma_{11}+\sigma_{21}\right)^{2} m^{2}+\left(\sigma_{12}+\sigma_{22}+g\right)^{2} m^{2} .
$$

In order to ensure $u \leq \bar{u}$, where $\bar{u}$ is a thrust bounded constant defined by the properties of the actuators, and from (61), then the following condition is necessary

$\left(\sigma_{11}+\sigma_{21}\right)^{2}+\left(\sigma_{12}+\sigma_{22}+g\right)^{2} \leq \frac{u^{2}}{m^{2}}$.

To guarantee the orientation angle $\theta$ tracks its desired value $\theta_{d}$ in the sense that $\lim _{t \rightarrow \infty}\left\|\theta(t)-\theta_{d}(t)\right\|=0$, such that

$\lim _{t \rightarrow \infty}\|\omega(t)\|=0$

Let us propose a Proportional Derivative controller with the form

$u_{2}=-k_{\theta}\left(\theta-\theta_{d}\right)-k_{\dot{\theta}}\left(\dot{\theta}-\dot{\theta_{d}}\right)$

where $k_{\theta}$ and $k_{\dot{\theta}}$ are positive constants that must be well chosen to ensure (63). $\dot{\theta_{d}}$ rate is defined as

$\dot{\theta}_{d}=\frac{\dot{F}_{1} F_{2}-F_{1} \dot{F}_{2}}{F_{2}^{2}+F_{1}^{2}}$

with

$$
\begin{aligned}
\dot{F}= & -\frac{1}{m}\left(\left(1-\sigma_{1} \tanh \left(A p_{1}+B p_{2}\right)^{2}\right) A p_{2}\right. \\
& +B\left(\left(1-\sigma_{1} \tanh \left(A p_{1}+B p_{2}\right)^{2}\right)\right. \\
& \left.\left.+\left(1-\sigma_{2} \tanh \left(B p_{2}\right)^{2}\right)\right) \dot{p}_{2}\right) .
\end{aligned}
$$

\section{Simulations and experimental results}

This section presents the simulation and experimental results to validate the control strategies developed in (15), (26), (39), (49). The scenario consists in moving the longitudinal axis of the vehicle as following: the vehicle starts at $x(0)=-2 m$ and $z(0)=1 m$, then, three set-points defined by $x_{r_{1}}=2, x_{r_{2}}=4$ and $x_{r_{3}}=5$ in meters are imposed as desired values.

For better illustrating the graphs obtained in simulation and experimental results, we denote as $L B$, $N L B, N S$, the Linear/NonLinear Backstepping and the Nested Saturation controllers, respectively, called also classical approaches. The HSC means the Hyperbolic Saturation Controller based on the fully actuated approach, named also virtual method. Parameters for

\begin{tabular}{|c|c|c|c|}
\hline Controller & Control parameters & Sim. Values & Exp. Values \\
\hline \multirow{4}{*}{$\mathrm{LB}$} & $k_{1}$ & 1 & 1.31 \\
\hline & $k_{2}$ & 1.2 & 1 \\
\hline & $k_{3}$ & 0.8 & 0.8 \\
\hline & $k_{4}$ & 0.1 & 0.1 \\
\hline \multirow{4}{*}{ NLB } & $\bar{k}_{1}$ & 0.04 & 0.04 \\
\hline & $\bar{k}_{2}$ & 0.035 & 0.0025 \\
\hline & $\bar{k}_{3}$ & 0.4 & 0.4 \\
\hline & $\bar{k}_{4}$ & 0.1 & 0.1 \\
\hline \multirow{4}{*}{$\mathrm{NS}$} & $a$ & 0.15 & 0.15 \\
\hline & $b$ & 0.22 & 0.22 \\
\hline & $c$ & 0.1 & 0.1 \\
\hline & $d$ & 0.3 & 0.3 \\
\hline \multirow{8}{*}{$\mathrm{HSC}$} & $\sigma_{1}$ & 0.5 & 0.5 \\
\hline & $\sigma_{2}$ & 0.1 & 0.2 \\
\hline & $\sigma_{3}$ & 0.5 & 0.5 \\
\hline & $\sigma_{4}$ & 0.35 & 0.4 \\
\hline & $a_{11}$ & 5 & 1 \\
\hline & $a_{22}$ & 0.9 & 1.3 \\
\hline & $b_{11}$ & 3 & 0.3 \\
\hline & $b_{22}$ & 0.55 & 0.6 \\
\hline
\end{tabular}
numerical and practical validation are shown in Table 1 .

Table 1: Parameters values used in the control laws in (15), (26), (39) and (49) used in simulation and experimental validation.

\subsection{Simulations}

The performance of the PVTOL vehicle when using controllers (15), (26), (39) and (49) tracking the setpoints previously defined is shown in Figures 2 and 4. Note from Figure 2 that, the convergence using con- 
trollers without bounded functions are faster than the others that use saturation functions. This performance is 'normal' because the saturation functions are chosen so small to guarantee a convergence from any initial condition. To increase the speed of convergence of these controllers, the bound of the saturation functions must be increased. Besides, as depicted in Figure 3, the $\theta$ angle responses for controllers NS and HSC are bounded, even if initial conditions are far from the desired position. In addition, for the controllers LB and NLB the angular position is bigger than for the NS and HSC controllers and its magnitude is related with the error position. Notice that only for the HSC algorithm the angular position tracks the imposed reference.

Figure 4 is the most important graph to analyze the performance of the four controllers. In this figure $z$ performance is depicted showing the advantage of using virtual controller from the classical one. Observe here that the controllers using classical procedure their altitude performance is degraded when tracking the references. In practical validation, this is observed as a drop in the altitude performance ( $z$ decreases). This drop is related to how far the desired value is. Similarly, the performance of the vehicle comparing the four control algorithms in the $x-z$ plane is displayed in Figure 5 . In one hand, it can be verified that when using control algorithms based on classical method, the behaviour of the vehicle in the $z$-axis is compromized. On the other hand, controllers based on the virtual approach compensate this error by means of the correction term $F_{2}$ in the total thrust.

In Figures 6 and 7 the control input responses for the four controllers are depicted. Notice from Figure 6

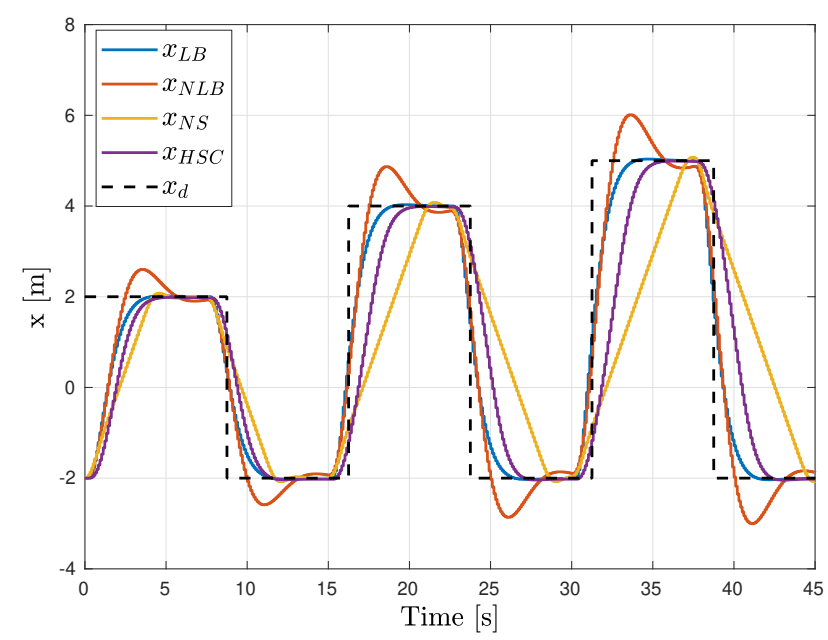

Fig. 2: Longitudinal position performance when comparing the four control algorithms.
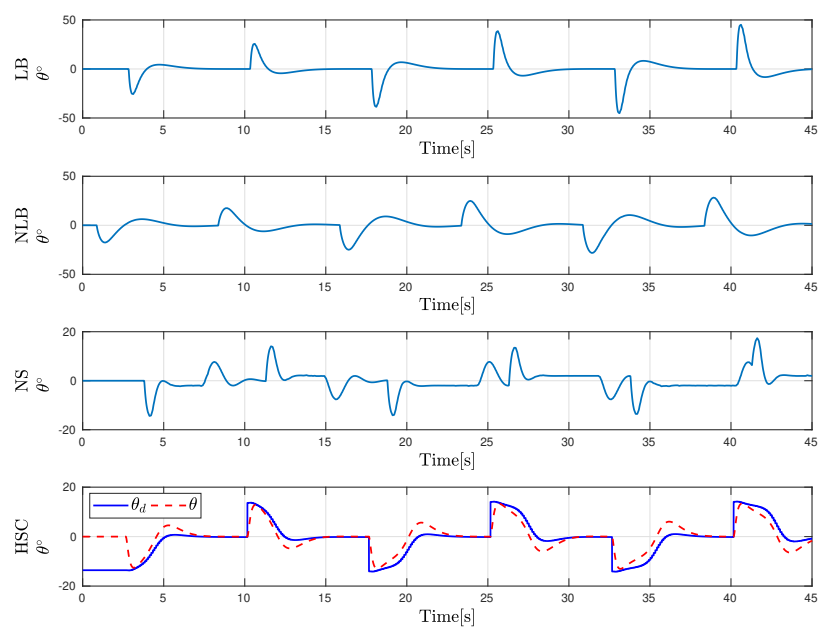

Fig. 3: $\theta$ angle response when applying four controllers into the PVTOL dynamics. $\theta_{d}$ denotes the desired angle computed in (44).

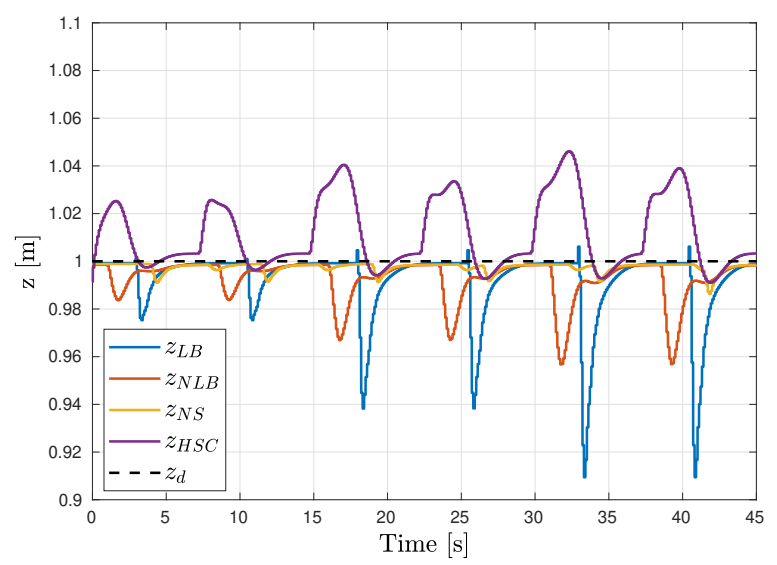

Fig. 4: Vertical position behavior when comparing the four control algorithms.

that $u_{1}$ response is different for the HSC controller with respect to the others that decreases when the desired point is modified. Notice that for the HSC controller $u_{1}$ increases to compensate the lateral displacement. Nevertheless, in Figure 7 the $u_{2}$ performance is quite different. Notice here that $u_{2}$ for the HSC controller is smaller with respect to the others one.

4.2 Experimental results

The control algorithms (15), (26), (39) and (49) were validated in real time in a quadcopter vehicle evolving as a PVTOL vehicle. For this, front motors $\left(f_{L_{2}}, f_{R_{2}}\right)$ and rear motors $\left(f_{L_{1}}, f_{R_{1}}\right)$ in the aerial vehicle are considered producing only a force in the front and rear side of the vehicle, as depicted in Figure 8. 


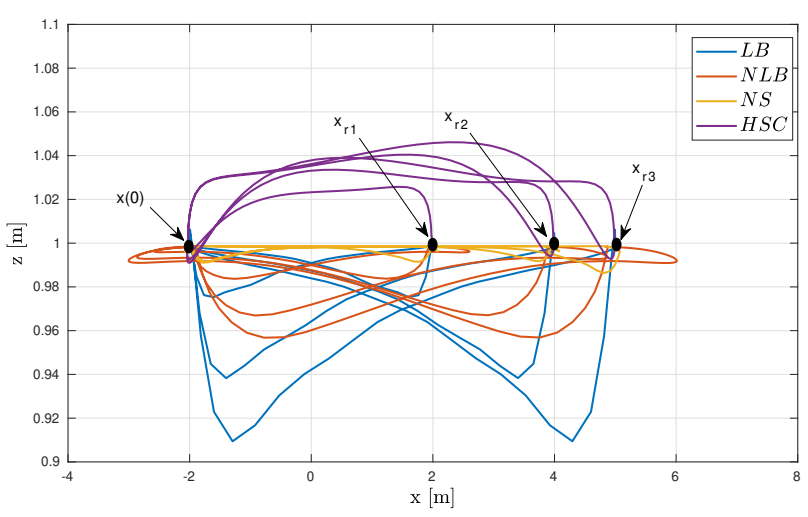

Fig. 5: Vehicle's performance evolution in the $x-z$ plane when applying the four control algorithms into the PVTOL dynamics. From figure $x_{r_{i}}$ for $i: 1,2,3$ describes the desired way-point.

From Figure 8 notice that $f_{1}=f_{L_{1}}+f_{R_{1}}$ and $f_{2}=f_{L_{2}}+f_{R_{2}}$. Vertical displacement on the $z$ axis is produced when increasing or decreasing, by the same magnitude the speed in the motors, while pitch moment is produced with the difference between $f_{1}$ and $f_{2}$.

This quadcopter vehicle evolving as a PVTOL is an AR Drone 2. Its firmware was modified to work under the software Fl-AIR - Framework libre AIR which is open source and runs a Linux-based operating system, capable of implementing a wide range of control schemes, see [21]. An OptiTrack motion capture system was used to estimate the vehicles position, while its internal Inertial Measurement Unit (IMU) measures its orientation and angular rates.

Conditione for flicht tacte aro the camo nood in cim-
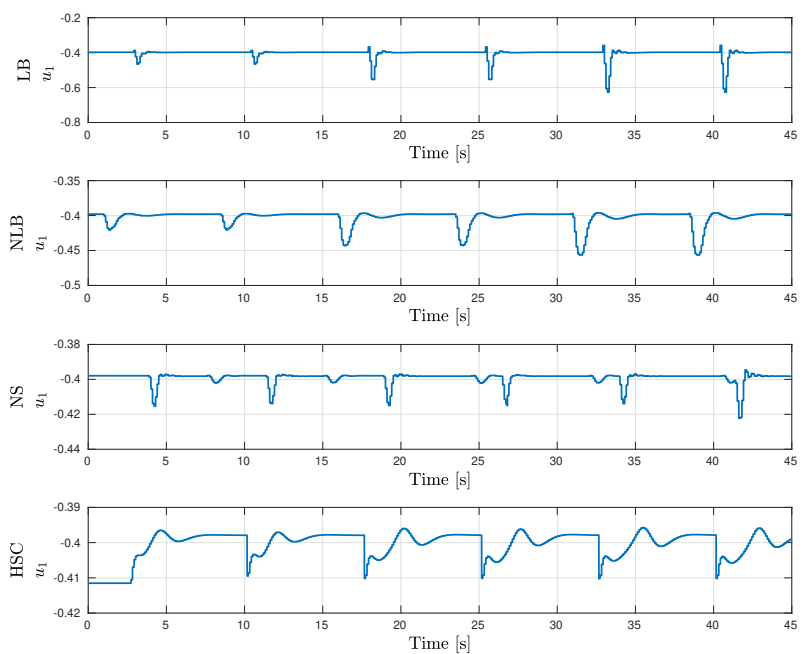

Fig. 6: $u_{1}$ behavior when applying the controllers (15), (26), (39) and (49).
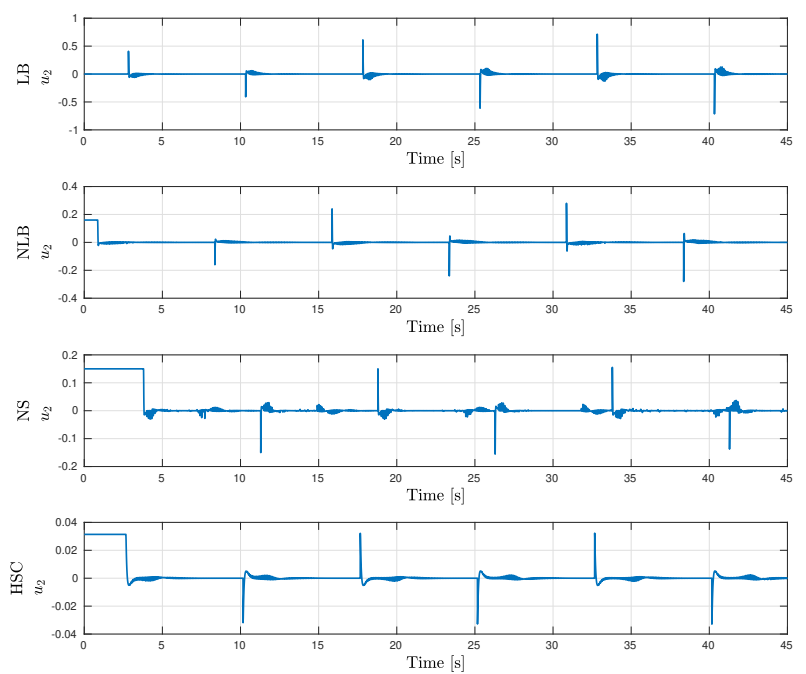

Fig. 7: Torque control $u_{2}$ response when applying the four control algorithms.

$z(0)=1 m$ while $x_{r_{1}}=2, x_{r_{2}}=4$ and $x_{r_{3}}=5$ in meters are the references. In Figures 9- 9 the state response when applying the controllers are illustrated.

Notice from Figure 9, as in the simulation case, the convergence of controllers based on saturation functions are quite slower due to the bound of these functions, that helps to maintain an relative small error with respect to the others controllers.

The angular performance of the system is depicted in Figure 11. Notice here that for the HSC algorithm a desired angle is imposed that is tracked satisfactory. Similarly in this figure observe that $\theta$-response is bigger for the other controllers.

Observe in Figure 11, that the magnitude of the error in the $z$-axis is directly related to the $x$-axis error.

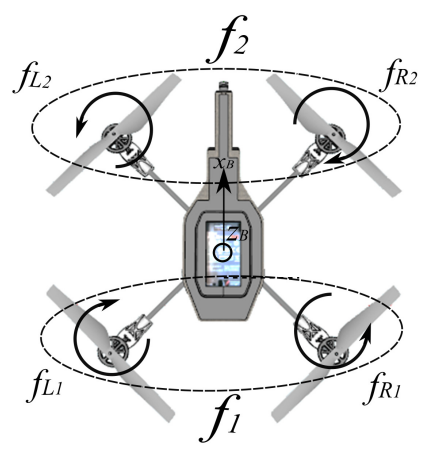

Fig. 8: Quadcopter configuration evolving in its longitudinal plane with the main forces acting in the vehicle. 


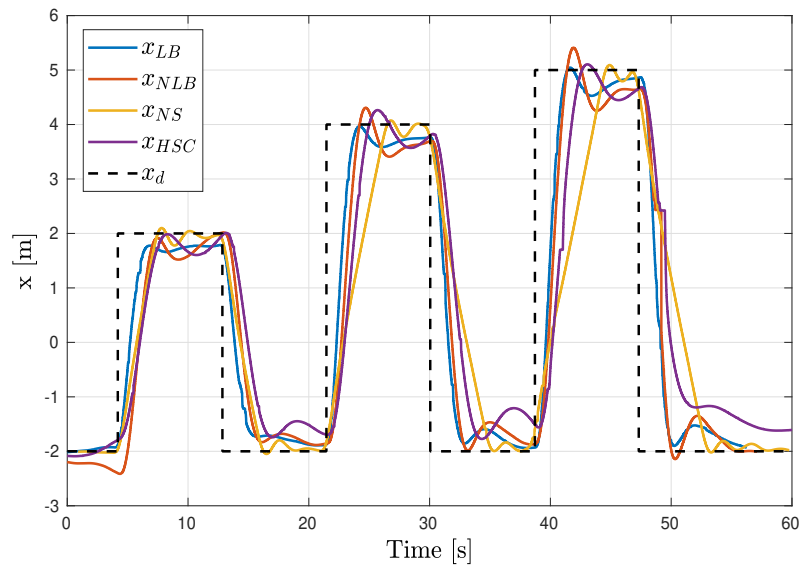

Fig. 9: Longitudinal behavior when applying experimentally the four control algorithms into the aerial vehicle.
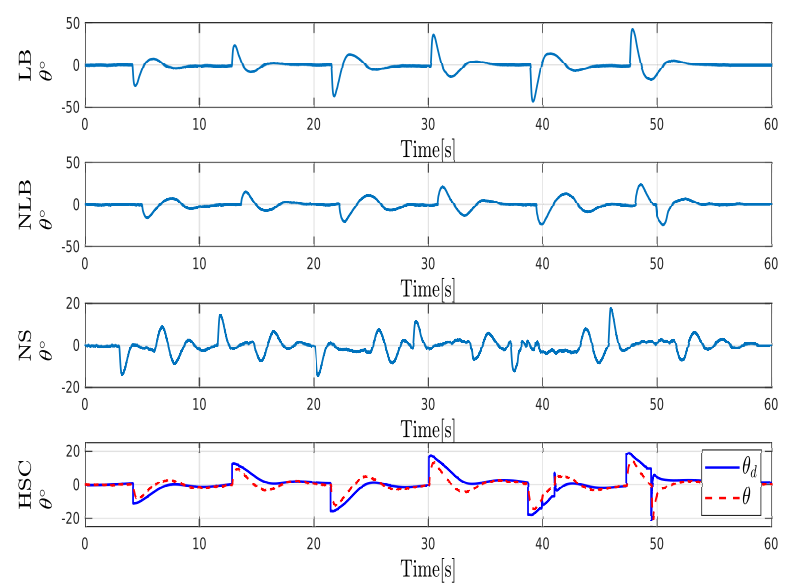

Fig. 10: $\theta$ angle response when the four control algorithms where applied in real time. $\theta_{d}$ describes the desired angle.

This means that if the desired $x$-position is far, then, the error in the vertical axis increases. And as a consequence, the magnitude of the $\theta$ angle is affected as can be verified in Figure 10. Therefore, as can be observed in Figure 10, it is possible to use small values for the saturation functions, nevertheless it will compromise the performance of the system.

Moreover, a big angle response should increase the vertical error in the vehicle when using control algorithms based on classical approach as can be seen in Figure 12a. Besides, when using controllers based on the virtual approach or controllers using bounded functions, this error is minimized as shown in Fig. 12b.
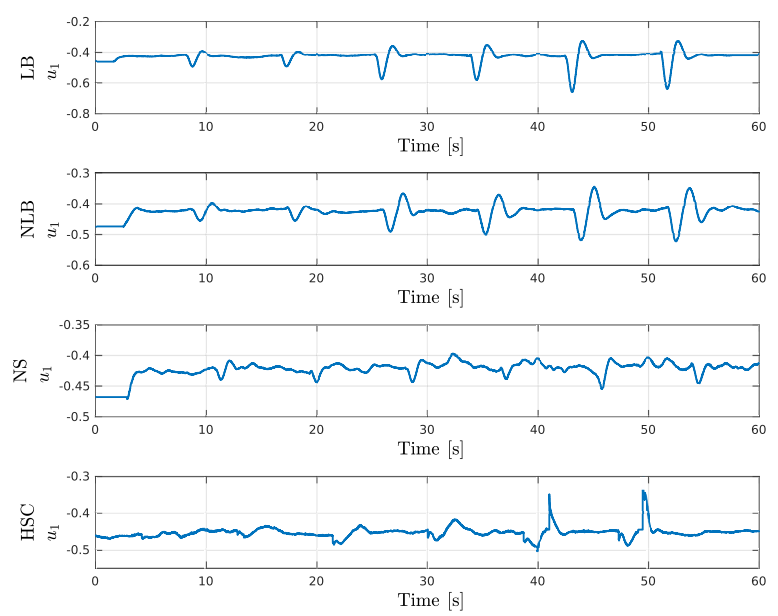

Fig. 13: Thrust control $u_{1}$ response for the four control algorithms applied to the aerial vehicle.

In Figures 13 and 14 the control input responses are illustrated. Notice that they have similar performance obtained in simulations.

\section{Discussion}

We have carried out an analysis of the performance of the four control algorithms. For this purpose, the performance indices and a qualitative comparison of the controller are obtained. The performance indices are the Integral Square Error (ISE), the Integral Absolute Error (IAE), the Integral time Squared Error (ITSE) and the Integral time Absolute Error (ITAE). A summary of this analysis is presented in Tables $2-4$.

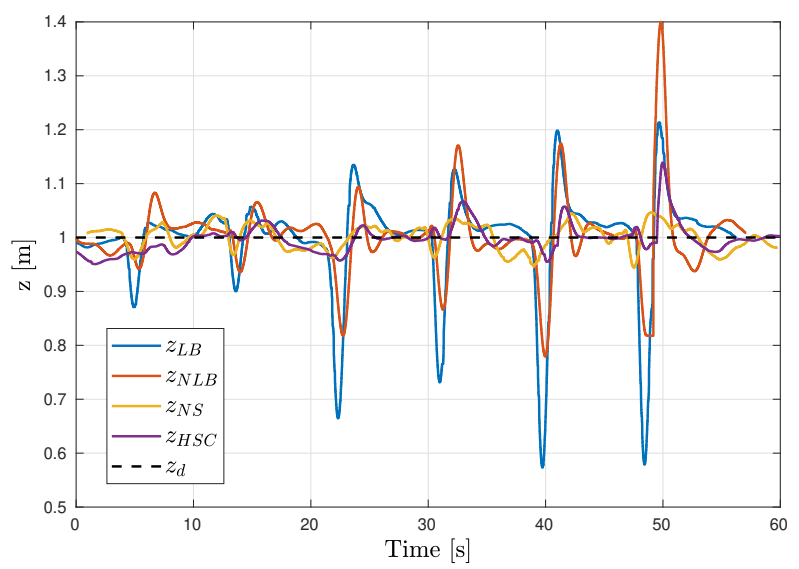

Fig. 11: Vertical position comparing the four control algorithms. 


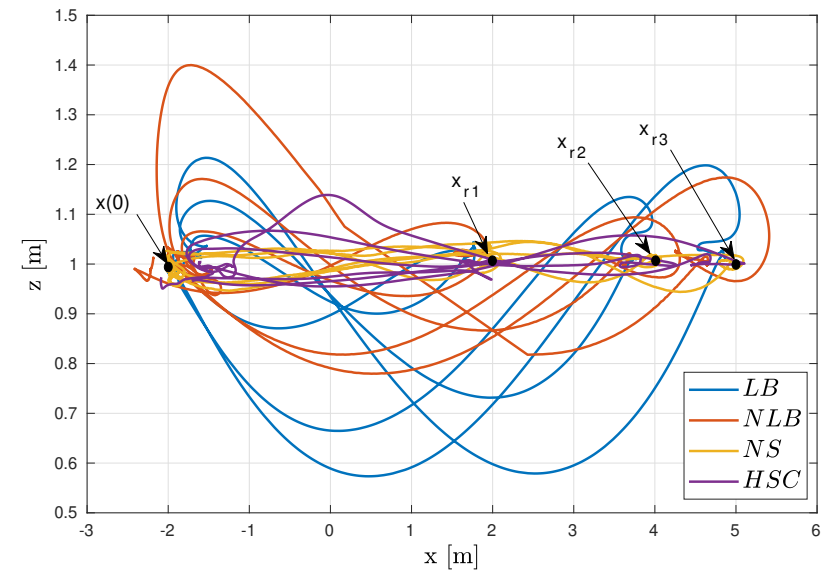

(a) Vehicle's performance evolution in the $x-z$ plane when the four control algorithms are applied in real time.

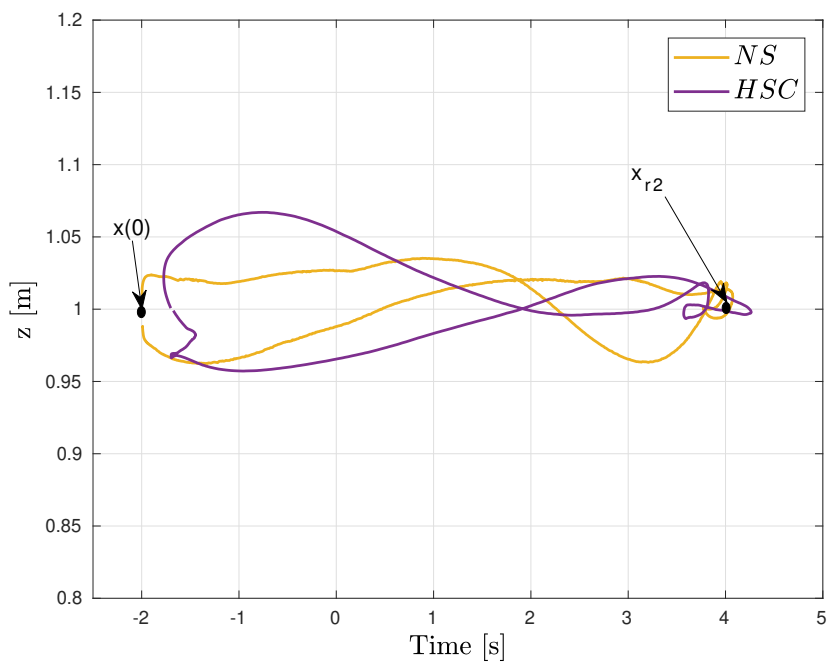

(b) $x z$ performance for the NS and HSC control algorithms.

Fig. 12: Vehicle's performance evolution in the $x-z$ plane comparing the four control algorithms.

From the steady state regime experimental results, in Table 2 it can be observed that the Nested Saturation and Hyperbolic Saturation controllers achieve the best performance in terms of the vertical error. The HSC presents less error than NS. However, the HSC algorithm includes a bigger computational effort with respect to the other algorithms due the derivatives of the desired angle $\theta_{d}$. It can be analyzed from the design of the HSC controller that it imposes desired angles, therefore, this increases the time of convergence to the desired translational values. Nevertheless, it could be an advantage because when imposing desired angles, we can ensure converge even if the desired position are far away. Observe also from tables that the Nested Saturation controller is quite small with respect to the other
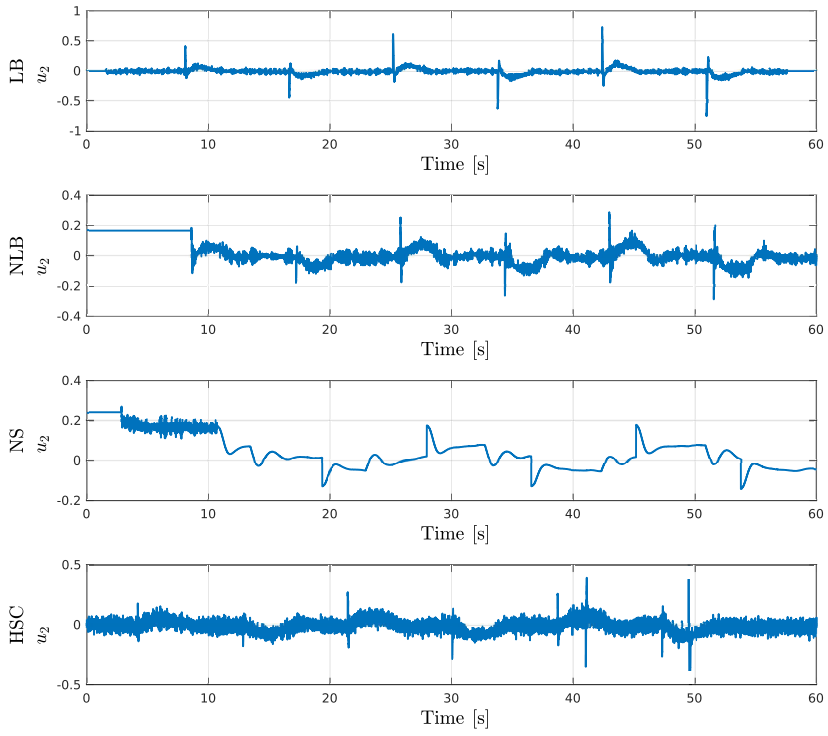

Fig. 14: Torque control response, $u_{2}$, obtained during the flight tests.

Table 2: Performance indices of the $z$-axis

\begin{tabular}{ccccc}
\hline Controllers/Indices & IAE & ISE & ITAE & ITSE \\
\hline LB & 2.97 & 0.48 & 99.48 & 17.98 \\
NLB & 2.40 & 0.30 & 82.38 & 12.33 \\
NS & 1.16 & 0.03 & 35.63 & 1.02 \\
HSC & 1.09 & 0.04 & 30.04 & 1.35 \\
\hline
\end{tabular}

approaches due mainly to the values of the bound of the saturation functions.

Table 3: Performance indices of the $x$-axis

\begin{tabular}{ccccc}
\hline Controllers/Indices & IAE & ISE & ITAE & ITSE \\
\hline LB & 60.21 & 221.56 & 1453 & 7132 \\
NLB & 64.53 & 241.37 & 1937 & 7780 \\
NS & 77.96 & 290.13 & 2324 & 10188 \\
HSC & 83.51 & 308.68 & 266.76 & 14752 \\
\hline
\end{tabular}

The linear backstepping controller presents a small error in the longitudinal axis and faster convergence time as can be observed in tables. Nonetheless, according to Table 2 this control algorithm has a bigger vertical error and by consequence control effort $u_{2}$ required is also bigger.

Regarding the design, tuning and the implementation effort, the linear backstepping and nested saturation algorithms are easier to tune because their structure can be seen as Proportional Derivative controllers. In contrast, the NLB and the HSC are tedious to implement since both present several complex derivatives 
and they have more parameters to tune. In this context, Table 4 presents a behavior comparison between the four algorithms including numerical and practical validation and other qualitative indicators.

Concerning the methods to design these control laws, one of the advantages of the virtual method is the possibility to design any control law (adaptive control, sliding mode control, etc) in $F_{1}$ and $F_{2}$ that could significantly improve the performance of the system. Here, the challenge will be to design control laws capable to provide desired angles respecting the mechanical constraints of the vehicle for avoiding singularities in the control input $u_{2}$. The disadvantage, as previously mentioned, is the complexity of the implementation of the algorithms due of the first and second derivative of $F_{1}$ and $F_{2}$.

Table 4: Qualitative comparison of the control algorithms. The best result is denoted with 1 and the worst with 4 .

\begin{tabular}{ccccc}
\hline & LB & NLB & NS & HSC \\
\hline Converge to & 1 & 4 & 3 & 2 \\
the reference & 1 & 3 & 2 & 3 \\
Computational effort & 1 & 3 & 1 & 1 \\
Control effort & 4 & 4 & 2 & 3 \\
Design \& tuning effort & 1 & 4 & 3 & 3 \\
Real implementation & 1 & 4 &
\end{tabular}

In contrast, the classical method gives the advantage of an easy fashion to get the analysis and design of the control algorithms based on the assumption that the vertical axis is controlled. However, the control effort of the inputs $u_{1}$ and $u_{2}$ could be bigger if the desired positions are far from the initial conditions.

\section{Conclusion}

This paper deals with a brief overview evaluation of popular control algorithms for VTOLs vehicles. Our goal was to provide a unified and accessible analysis, placing the classical model of a multirotor aerial vehicle and the proposed control methods (Classical and Virtual) into a proper context. In this case, four control algorithms were obtained, explaining the main procedure for their design. The studied control methodologies were the linear and nonlinear backsteping and the nested saturation functions for the well-known underactuated dynamics and the hyperbolic saturation control for a virtual fully actuated system. The Lyapunov theory was used to prove the stability analysis.
Simulation and experimental results revealed that using the virtual approach for the control design results in a smoother convergence to the desired reference and a smaller control effort demand. After analysis, we consider that virtual approach should be a good solution for some mission and need to be explored in more detail. Nevertheless, the choice between the controllers will depend on the problem requirements.

Acknowledgements This work was supported by CONACyT (Consejo Nacional de Ciencia y Tecnología), Mexico. This work has been also sponsored by the French government research programm Investissements davenir through the Robotex Equipment of Excellence (ANR-10-EQPX-44). Theirs supports are gratefully acknowledge

\section{Compliance with Ethical Standards}

Conflict of interests The authors declare that they have no conflict of interest.

\section{References}

1. Abaunza, H., Ibarra, E., Castillo, P., Victorino, A. Quaternion based control for circular uav trajectory tracking, following a ground vehicle: Real-time validation. IFAC-PapersOnLine 50(1), 11453 - 11458 (2017). DOI 10.1016/j.ifacol.2017.08.1816. 20th IFAC World Congress

2. Ailon, A.: Simple tracking controllers for autonomous vtol aircraft with bounded inputs. IEEE Transactions on Automatic Control 55(3), 737-743 (2010). DOI 10.1109/TAC.2010.2040493

3. Amin, R., Aijun, L., Shamshirband, S.: A review of quadrotor uav: control methodologies and performance evaluation. International Journal of Automation and Control 10(2), 87-103 (2016). DOI 10.1504/IJAAC.2016. 076453. PMID: 76453

4. Ansari, U., Bajodah, A.H.: Tracking control of quadrotor using generalized dynamic inversion with constantproportional rate reaching law. In: 2019 1st International Conference on Unmanned Vehicle Systems-Oman (UVS), pp. 1-7 (2019). DOI 10.1109/UVS.2019.8658292

5. Antonio-Toledo, M.E., Sanchez, E.N., Alanis, A.Y., Flrez, J., Perez-Cisneros, M.A.: Real-time integral backstepping with sliding mode control for a quadrotor uav. IFAC-PapersOnLine 51(13), 549 - 554 (2018). DOI 10.1016/j.ifacol.2018.07.337. 2nd IFAC Conference on Modelling, Identification and Control of Nonlinear Systems MICNON 2018

6. Beikzadeh, H., Liu, G.: Trajectory tracking of quadrotor flying manipulators using 11 adaptive control. Journal of the Franklin Institute 355(14), 6239 - 6261 (2018). DOI 10.1016/j.jfranklin.2018.06.011

7. Castillo, P., Lozano, R., Dzul, A.E.: Modelling and Control of Mini-Flying Machines. Springer-Verlag London (2006). DOI 10.1007/1-84628-179-2

8. Chen, F., Lu, F., Jiang, B., Tao, G.: Adaptive compensation control of the quadrotor helicopter using quantum 
information technology and disturbance observer. Journal of the Franklin Institute 351(1), 442 - 455 (2014). DOI 10.1016/j.jfranklin.2013.09.009

9. Das, A., Subbarao, K., Lewis, F.: Dynamic inversion with zero-dynamics stabilisation for quadrotor control. IET Control Theory Applications 3(3), 303-314 (2009). DOI 10.1049/iet-cta:20080002

10. de Crousaz, C., Farshidian, F., Neunert, M., Buchli, J.: Unified motion control for dynamic quadrotor maneuvers demonstrated on slung load and rotor failure tasks. In: 2015 IEEE International Conference on Robotics and Automation (ICRA), pp. 2223-2229 (2015). DOI 10.1109/ICRA.2015.7139493

11. Escareño, J., Salazar, S., Romero, H., Lozano, R.: Trajectory control of a quadrotor subject to $2 \mathrm{~d}$ wind disturbances. Journal of Intelligent \& Robotic Systems 70(1), 51-63 (2013). DOI 10.1007/s10846-012-9734-1

12. Faessler, M., Falanga, D., Scaramuzza, D.: Thrust Mixing, Saturation, and Body-Rate Control for Accurate Aggressive Quadrotor Flight. IEEE Robotics and Automation Letters 2(2), 476-482 (2017). DOI 10.1109/LRA. 2016.2640362

13. Faessler, M., Franchi, A., Scaramuzza, D.: Differential flatness of quadrotor dynamics subject to rotor drag for accurate tracking of high-speed trajectories. IEEE Robotics and Automation Letters 3(2), 620-626 (2018). DOI 10.1109/LRA.2017.2776353

14. Gandolfo, D.C., Salinas, L.R., Brando, A., Toibero, J.M.: Stable path-following control for a quadrotor helicopter considering energy consumption. IEEE Transactions on Control Systems Technology 25(4), 1423-1430 (2017). DOI 10.1109/TCST.2016.2601288

15. Guerrero-Castellanos, J., Marchand, N., Hably, A., Lesecq, S., Delamare, J.: Bounded attitude control of rigid bodies: Real-time experimentation to a quadrotor mini-helicopter. Control Engineering Practice 19(8), 790 - 797 (2011). DOI 10.1016/j.conengprac.2011.04.004

16. Hehn, M., D'Andrea, R.: Real-time trajectory generation for interception maneuvers with quadrocopters. In: 2012 IEEE/RSJ International Conference on Intelligent Robots and Systems, pp. 4979-4984 (2012). DOI 10.1109/IROS.2012.6386093

17. Heredia, G., Jimenez-Cano, A.E., Sanchez, I., Llorente, D., Vega, V., Braga, J., Acosta, J.A., Ollero, A.: Control of a multirotor outdoor aerial manipulator. In: 2014 IEEE/RSJ International Conference on Intelligent Robots and Systems, pp. 3417-3422 (2014). DOI 10.1109/IROS.2014.6943038

18. Ibarra-Jimenez, E., Castillo, P.: Aerial autonomous catching ball using a nested second order sliding mode control. IFAC-PapersOnLine 50(1), 11415 - 11420 (2017). DOI 10.1016/j.ifacol.2017.08.1805. 20th IFAC World Congress

19. Kara-Mohamed, M.: Advanced trajectory tracking for uavs using combined feedforward/feedback control design. Robotics and Autonomous Systems 96, 143 - 156 (2017). DOI 10.1016/j.robot.2017.07.009

20. Khalil, H.K.: Nonlinear systems (3rd ed. ). Upper Saddle River, NJ: Prentice Hall (2002)

21. Lab, H.: Fl-air - framework libre air (2012). URL https: //devel.hds.utc.fr/software/flair

22. L'Afflitto, A., Anderson, R.B., Mohammadi, K.: An introduction to nonlinear robust control for unmanned quadrotor aircraft: How to design control algorithms for quadrotors using sliding mode control and adaptive control techniques. IEEE Control Systems Magazine 38(3), 102-121 (2018). DOI 10.1109/MCS.2018.2810559
23. Lee, H., Kim, H.J.: Trajectory tracking control of multirotors from modelling to experiments: A survey. International Journal of Control, Automation and Systems 15(1), 281-292 (2017). DOI 10.1007/s12555-015-0289-3

24. Lei, W., Li, C., Chen, M.Z.Q.: Robust adaptive tracking control for quadrotors by combining pi and self-tuning regulator. IEEE Transactions on Control Systems Technology 27(6), 2663-2671 (2019). DOI 10.1109/TCST. 2018.2872462

25. Loianno, G., Brunner, C., McGrath, G., Kumar, V.: Estimation, Control, and Planning for Aggressive Flight With a Small Quadrotor With a Single Camera and IMU. IEEE Robotics and Automation Letters 2(2), 404-411 (2017). DOI 10.1109/LRA.2016.2633290

26. Lu, H., Liu, C., Coombes, M., Guo, L., Chen, W.: Online optimisation-based backstepping control design with application to quadrotor. IET Control Theory Applications 10(14), 1601-1611 (2016). DOI 10.1049/iet-cta. 2015.0976

27. Ma, D., Xia, Y., Shen, G., Jia, Z., Li, T.: Flatness-based adaptive sliding mode tracking control for a quadrotor with disturbances. Journal of the Franklin Institute 355(14), 6300 - 6322 (2018). DOI 10.1016/j.jfranklin. 2018.06.018

28. Mac, T.T., Copot, C., Keyser, R.D., Ionescu, C.M.: The development of an autonomous navigation system with optimal control of an uav in partly unknown indoor environment. Mechatronics 49, $187-196$ (2018). DOI 10.1016/j.mechatronics.2017.11.014

29. Mellinger, D., Kumar, V.: Minimum snap trajectory generation and control for quadrotors. In: 2011 IEEE International Conference on Robotics and Automation, pp. 2520-2525 (2011). DOI 10.1109/ICRA.2011.5980409

30. Mian, A.A., Daobo, W.: Modeling and backsteppingbased nonlinear control strategy for a 6 dof quadrotor helicopter. Chinese Journal of Aeronautics 21(3), 261 268 (2008). DOI 10.1016/S1000-9361(08)60034-5

31. Nascimento, T.P., Saska, M.: Position and attitude control of multi-rotor aerial vehicles: A survey. Annual Reviews in Control 52(7), 1367-5788 (2019)

32. Nayak, V., Karaya, R.R.: Target tracking by a quadrotor using proximity sensor fusion based on a sigmoid function. IFAC-PapersOnLine 51(1), 154 - 159 (2018). DOI 10.1016/j.ifacol.2018.05.026. 5th IFAC Conference on Advances in Control and Optimization of Dynamical Systems ACODS 2018

33. Nazaruddin, Y.Y., Andrini, A.D., Anditio, B.: Pso based pid controller for quadrotor with virtual sensor. IFACPapersOnLine 51(4), 358 - 363 (2018). DOI 10.1016/j. ifacol.2018.06.091. 3rd IFAC Conference on Advances in Proportional-Integral-Derivative Control PID 2018

34. Olfati-Saber, R.: Global configuration stabilization for the vtol aircraft with strong input coupling. IEEE Transactions on Automatic Control 47(11), 1949-1952 (2002). DOI 10.1109/TAC.2002.804457

35. Purwin, O., DAndrea, R.: Performing and extending aggressive maneuvers using iterative learning control. Robotics and Autonomous Systems 59(1), 1 - 11 (2011). DOI 10.1016/j.robot.2010.09.004

36. Rosaldo-Serrano, M., Aranda-Bricaire, E.: Trajectory tracking for a commercial quadrotor via time-varying backstepping. IFAC-PapersOnLine 51(13), 532 - 536 (2018). DOI 10.1016/j.ifacol.2018.07.334. 2nd IFAC Conference on Modelling, Identification and Control of Nonlinear Systems MICNON 2018

37. Ros, H., Falcn, R., Gonzlez, O.A., Dzul, A.: Continuous sliding-mode control strategies for quadrotor robust 
tracking: Real-time application. IEEE Transactions on Industrial Electronics 66(2), 1264-1272 (2019). DOI 10.1109/TIE.2018.2831191

38. Sanahuja, G., Castillo, P., Sanchez, A.: Stabilization of n integrators in cascade with bounded input with experimental application to a vtol laboratory system. International Journal of Robust and Nonlinear Control 20(10), 1129-1139 (2010). DOI 10.1002/rnc.1494

39. Santiaguillo-Salinas, J., Rosaldo-Serrano, M., ArandaBricaire, E.: Observer-based time-varying backstepping control for parrots ar.drone 2.0. IFAC-PapersOnLine 50(1), 10305 - 10310 (2017). DOI 10.1016/j.ifacol.2017. 08.1497. 20th IFAC World Congress

40. Shraim, H., Awada, A., Youness, R.: A survey on quadrotors: Configurations, modeling and identification, control, collision avoidance, fault diagnosis and tolerant control. IEEE Aerospace and Electronic Systems Magazine 33(7), 14-33 (2018). DOI 10.1109/MAES.2018.160246

41. Subudhi, C.S., Ezhilarasi, D.: Modeling and trajectory tracking with cascaded pd controller for quadrotor. Procedia Computer Science 133, 952 - 959 (2018). DOI 10.1016/j.procs.2018.07.082. International Conference on Robotics and Smart Manufacturing (RoSMa2018)

42. Tamayo, A.J.M., Ros, C.A.V., Zannatha, J.M.I., Soto, S.M.O.: Quadrotor input-output linearization and cascade control. IFAC-PapersOnLine" 51(13), 437 - 442 (2018). DOI 10.1016/j.ifacol.2018.07.317. 2nd IFAC Conference on Modelling, Identification and Control of Nonlinear Systems MICNON 2018

43. Teel, A.R.: Global stabilization and restricted tracking for multiple integrators with bounded controls. Systems \& Control Letters 18(3), 165 - 171 (1992). DOI 10.1016/ 0167-6911(92)90001-9

44. Tomashevich, S., Belyavskyi, A.: Passification based simple adaptive control of quadrotor. IFAC-PapersOnLine 49(13), 281 - 286 (2016). DOI 10.1016/j.ifacol.2016.07. 974. 12th IFAC Workshop on Adaptation and Learning in Control and Signal Processing ALCOSP 2016

45. Tomi, T., Maier, M., Haddadin, S.: Learning quadrotor maneuvers from optimal control and generalizing in real-time. In: 2014 IEEE International Conference on Robotics and Automation (ICRA), pp. 1747-1754 (2014). DOI 10.1109/ICRA.2014.6907087

46. Tran, T.T., Shuzhi Sam, G., Wei, H.: Adaptive control of a quadrotor aerial vehicle with input constraints and uncertain parameters. International Journal of Control (2018)

47. Villagmez, J., Vargas, M., Ortega, M., Rubio, F.: Modeling and control of the pvtol. IFAC-PapersOnLine 48(9), $150-155$ (2015). DOI 10.1016/j.ifacol.2015.08.075

48. Wang, R., Liu, J.: Trajectory tracking control of a 6-dof quadrotor uav with input saturation via backstepping. Journal of the Franklin Institute 355(7), 3288 - 3309 (2018). DOI 10.1016/j.jfranklin.2018.01.039

49. Warier, R.R., Sanyal, A.K., Dhullipalla, M.H., Viswanathan, S.P.: Trajectory tracking control for underactuated thrust-propelled aerial vehicles. IFACPapersOnLine 51(13), 555 - 560 (2018). DOI 10.1016/j.ifacol.2018.07.338. 2nd IFAC Conference on Modelling, Identification and Control of Nonlinear Systems MICNON 2018

50. Xu, Z., Nian, X., Wang, H., Chen, Y.: Robust guaranteed cost tracking control of quadrotor uav with uncertainties. ISA Transactions 69, 157 - 165 (2017). DOI 10.1016/j. isatra.2017.03.023

51. Yao, X., Chen, Z.: Sliding mode control with deep learning method for rotor trajectory control of active magnetic bearing system. Transactions of the Institute of Measurement and Control 41(5), 1383-1394 (2019). DOI $10.1177 / 0142331218778324$

52. Zagaris, A., Kaper, H.G., Kaper, T.J.: Fast and Slow Dynamics for the Computational Singular Perturbation Method. arXiv Mathematics e-prints math/0401206 (2004)

53. Zhang, Y., Chen, Z., Zhang, X., Sun, Q., Sun, M.: A novel control scheme for quadrotor uav based upon active disturbance rejection control. Aerospace Science and Technology 79, 601 - 609 (2018). DOI 10.1016/j.ast.2018.06. 017

54. Zhao, B., Tang, Y., Wu, C., Du, W.: Vision-based tracking control of quadrotor with backstepping sliding mode control. IEEE Access 6, 72439-72448 (2018). DOI 10.1109/ACCESS.2018.2882241

55. zbek, N.S., nkol, M., nder Efe, M.: Feedback control strategies for quadrotor-type aerial robots: a survey. Transactions of the Institute of Measurement and Control 38(5), 529-554 (2016). DOI 10.1177/0142331215608427 